\title{
Uso do lodo de ETE na produção de tijolos cerâmicos: Uma revisão sistemática de
}

\section{literatura}

\author{
Use of WWTP sludge in ceramic brick production: A systematic literature review
}

\section{Utilización de lodos de EDAR en la producción de ladrillos cerámicos: Una revisión bibliográfica} sistemática

\author{
Recebido: 15/06/2021 | Revisado: 22/06/2021 | Aceito: 25/06/2021 | Publicado: 12/07/2021 \\ José Douglas dos Santos Siqueira Silva \\ ORCID: https://orcid.org/0000-0002-6955-5667 \\ Instituto Federal de Educação, Ciência e Tecnologia do Rio Grande do Norte, Brasil \\ E-mail: jose.douglas@ifrn.edu.br \\ Régia Lúcia Lopes \\ ORCID: https://orcid.org/0000-0001-7435-1258 \\ Instituto Federal de Educação, Ciência e Tecnologia do Rio Grande do Norte, Brasil \\ E-mail: regia.lopes@ifrn.edu.br \\ Dayana Melo Torres \\ ORCID: https://orcid.org/0000-0002-9385-3234 \\ Instituto Federal de Educação, Ciência e Tecnologia do Rio Grande do Norte, Brasil \\ E-mail: dayana.torres@ifrn.edu.br \\ Renato Dantas Rocha da Silva \\ ORCID: https://orcid.org/0000-0002-0937-9032 \\ Instituto Federal de Educação, Ciência e Tecnologia do Rio Grande do Norte, Brasil \\ E-mail: renato.dantas@ifrn.edu.br
}

\begin{abstract}
Resumo
O lodo gerado nas estações de tratamento de esgoto (ETE) é um desafio ambiental para várias cidades com relação à sua destinação. A indústria de tijolos cerâmicos tem potencial para reaproveitar esse resíduo, podendo ser então uma alternativa sustentável. Desta forma, este trabalho tem como proposta identificar na literatura a viabilidade técnica da aplicação do lodo de ETE diretamente no processo de fabricação do tijolo cerâmico em substituição parcial à argila. A metodologia utilizada foi uma revisão sistemática de literatura e os critérios P.I.C.O como estratégia de partida. Foram utilizados 4 critérios de exclusão na escolha dos artigos e chegou-se a 5 artigos científicos que abordavam a temática. Verificou-se dados relacionados aos parâmetros de composição química, proporção de mistura, temperatura de sinterização, perda de peso na ignição, densidade aparente, absorção de água, retração linear, resistência mecânica e lixiviação de metais sendo estes comparados, em cada estudo, aos limites normativos locais de fabricação de tijolos cerâmicos. Neste trabalho, a norma brasileira ABNT NBR 15270-1 também foi utilizada como comparativo aos dados verificados. Os resultados foram promissores, pois em cada trabalho apresentou-se ao menos uma proporção de mistura (traço) que indicasse um tijolo dentro dos limites normativos. Logo, conclui-se que é viável tecnicamente desenvolver tijolos com argila e lodo de ETE na sua constituição primária de fabricação.
\end{abstract}

Palavras-chave: Argila; Lodo de esgoto; Estação de tratamento de esgoto; Tijolo cerâmico.

\begin{abstract}
The sludge generated in wastewater treatment plant (WWTP) is a challenge for several cities about its destination. The ceramic brick industry has the potential to reuse this waste and can therefore be a sustainable alternative. Therefore, this work aims to identify in the literature if it is possible to apply the ETE sludge directly in the manufacturing process of the clay brick in partial substitution to the clay. The methodology used was a systematic literature review and the P.I.C.O. criteria as a starting strategy. Four exclusion criteria were used in the choice of articles and 5 scientific articles were arrived at that addressed the subject. Data related to chemical composition parameters, mixing ratio, sintering temperature, weight loss on ignition, apparent density, water absorption, linear shrinkage, mechanical resistance and metal leaching were verified, and these were compared, in each study, to the local normative limits of ceramic brick manufacture. In this work, the Brazilian standard ABNT NBR 15270-1 was also used as a comparison to the verified data. The results were promising, since in each work at least one proportion of the mixture (trace) indicating a brick within the normative limits was presented. Therefore, it is concluded that it is technically feasible to develop bricks with clay and WWTP sludge in their primary manufacture.
\end{abstract}

Keywords: Clay; Sewage sludge; Wastewater treatment plant; Ceramic brick. 


\begin{abstract}
Resumen
Los lodos generados en las estaciones depuradoras de aguas residuales (EDAR) suponen un reto medioambiental para varias ciudades en cuanto a su destino. La industria de los ladrillos cerámicos tiene el potencial de reutilizar estos residuos y puede ser entonces una alternativa sostenible. Así, este trabajo pretende identificar en la literatura la viabilidad técnica de aplicar los lodos de EDAR directamente en el proceso de fabricación de ladrillos cerámicos como sustitución parcial de la arcilla. La metodología utilizada fue una revisión sistemática de la literatura y los criterios de la O.I.P. como estrategia de partida. Se utilizaron cuatro criterios de exclusión en la elección de los artículos y se llegó a 5 artículos científicos que abordaban el tema. Se verificaron los datos relativos a los parámetros de composición química, proporción de la mezcla, temperatura de sinterización, pérdida de peso por ignición, densidad aparente, absorción de agua, contracción lineal, resistencia mecánica y lixiviación de metales y se compararon en cada estudio, con los límites normativos locales de fabricación de ladrillos cerámicos. En este trabajo también se utilizó la norma brasileña ABNT NBR 15270-1 como comparación con los datos verificados. Los resultados fueron prometedores, ya que en cada trabajo se presentó al menos una proporción de combinación (mezcla) que indicaba un ladrillo dentro de los límites normativos. Por lo tanto, se concluye que es técnicamente factible elaborar ladrillos con arcilla y lodos de depuradora en su constitución primaria de fabricación.
\end{abstract}

Palabras clave: Arcilla; Lodos de depuradora; Planta de tratamiento de aguas residuales; Ladrillo cerámico.

\title{
1. Introdução
}

A destinação do lodo das estações de tratamento de esgoto (ETE) é um grande desafio ambiental mundial (Cusidó \& Cremades, 2012). O lodo de ETE é um material semissólido subproduto dos processos de tratamento das águas residuárias municipais, oriundos de fontes residências, institucionais, comerciais e industriais, sendo então um resíduo final (Areias et al., 2020; Esmeray \& Atis, 2019; Feng et al., 2014; Kadir et al., 2019).

As estratégias das cidades com relação a destinação deste tipo de lodo são a incineração, destinação aos aterros, reúso na agricultura e até o lançamento em oceanos em alguns países (Liew et al., 2004; Montero et al., 2009; Smol et al., 2015).

O descarte de lodo de esgoto é um problema para os munícipios, devido ao potencial poluidor das substâncias presentes nesse resíduo, ao rigor das regulamentações ambientais e o crescimento industrial (Liew et al., 2004). Além disso, representa um custo relevante com o seu manejo para a gestão da ETE, ficando entre $20 \%$ e $60 \%$ do custo operacional de toda a estação (Uggetti et al., 2010). Em 2017, foram gerados cerca de 45 milhões de toneladas de lodo de ETE no mundo (Chang et al., 2020; Zhang et al., 2017). Espera-se que a quantidade de lodo gerada pelas cidades aumente à medida que cresça os investimentos e padrões ambientais com novas Estações de Tratamento de Esgoto (Esmeray \& Atis, 2019).

Para Martínez-García et al. (2012), o setor da construção civil é o mais indicado para buscar alternativas de reutilização desse resíduo por possuir uma variabilidade enorme de matérias-primas e produtos. Corroborando a isso, muitos estudos vêm sendo desenvolvidos com o uso direto do lodo de esgoto na fabricação de materiais de construção com o propósito de reciclar este tipo de resíduo e inseri-lo novamente no ciclo produtivo (Taki et al., 2020; Yang et al., 2021). No Japão, 48\% da quantidade de lodo de esgoto gerada no país já é reinserida nos processos de fabricação de materiais construtivos (Chang et al., 2020).

O lodo de ETE apresenta uma composição mineralógica próxima à argila, pois integra óxidos comuns aos dois materiais como $\mathrm{SiO}_{2}, \mathrm{Al}_{2} \mathrm{O}_{3}, \mathrm{CaO}$ e $\mathrm{Fe}_{2} \mathrm{O}_{3}$ (Chang et al., 2020). Desta forma, os materiais cerâmicos apresentam um destino promissor para integrar este tipo de lodo na composição da matéria-prima dos seus elementos (Martínez-García et al., 2012).

Logo, essa pesquisa visa identificar o potencial de aplicação direta do lodo oriundo de estações de tratamento de esgoto (ETE) em substituição parcial à argila no processo de fabricação de tijolos cerâmicos através de uma revisão sistemática de literatura.

\section{Metodologia}

Este trabalho adotou a metodologia da Revisão Sistemática de Literatura (RSL) para realizar o levantamento de informações sobre a utilização do lodo de Estação de Tratamento de Esgoto (ETE) na fabricação de tijolos cerâmicos por 
permitir uma busca mais refinada, de grande alcance e de maneira organizada (Koller et al., 2014). Com os resultados apresentados pela RSL, os dados podem ser facilmente analisados e validados (Souza et al., 2020).

De acordo com Koller et al. (2014), o método a ser seguido em uma RSL apresenta 8 etapas: (1) delimitação da questão de partida (2) escolha das bases de dados; (3) escolha das palavras-chave; (4) busca e armazenamento dos resultados; (5) seleção dos artigos pelo resumo e considerando os critérios de inclusão e exclusão; (6) extrair a informação dos artigos selecionados; (7) avaliação dos artigos; e (8) síntese e interpretação dos dados.

Para a delimitação da questão de partida, aplicou-se os critérios do método população, intervenção, comparação e resultado (P.I.C.O), conforme apresentado na Quadro 1, que viabilizaram a construção da ideia sobre a escolha das principais palavras-chave, focando no escopo do estudo e evitando perda de tempo com buscas desnecessárias (Santos et al., 2007; Souza et al., 2020).

Quadro 1 - Critérios P.I.C.O adotados para o levantamento de estudos.

\begin{tabular}{|l|l|}
\hline \multicolumn{1}{|c|}{ CRITÉRIOS P.I.C.O } & \multicolumn{1}{c|}{ DESCRIÇÃ̃O } \\
\hline POPULAÇÃO & Produção de tijolos cerâmicos \\
\hline INTERVENÇÃO & Com lodo de ETE \\
\hline COMPARAÇÃO & Com argila vermelha \\
\hline RESULTADO & Verificar a viabilidade técnica \\
\hline
\end{tabular}

Fonte: Autores (2020).

Para realização do levantamento de artigos, utilizou-se de duas bases de pesquisa: Sciencedirect e Scopus. Conforme os critérios estabelecidos no Quadro 1, as palavras-chave consideradas na busca dos trabalhos e associadas ao tema foram: tijolo cerâmico (ceramic brick), tijolo de argila (clay brick) e lodo de esgoto (sewage sludge). A partir disso, foi montada a seguinte string de busca: "sewage sludge" AND ("clay brick" OR ceramic brick).

Após a primeira pesquisa na base de dados com a string de busca adotada, foram utilizados 4 critérios de exclusão: $1^{\circ}$ - O período analisado foi de 2000 até 2021; $2^{\circ}$ - O tipo de artigo deveria ser Research articles (Sciencedirect) ou Articles (Scopus); $3^{\circ}$ - Somente artigos com o uso exclusivo (sem adição de outro tipo de material ou resíduos) de lodo de ETE e argila vermelha para a finalidade de fabricação em tijolos cerâmicos; $4^{\circ}$ - exclusão de artigos sem acesso. Após as etapas de exclusão, não foram encontrados artigos duplicados entre os selecionados das duas bases, resultando então em 5 artigos selecionados conforme a Quadro 2.

Quadro 2 - Seleção e busca dos artigos com base na string de busca.

\begin{tabular}{|c|c|c|c|c|c|c|c|}
\hline \multirow[t]{2}{*}{ BASE DE DADOS } & \multirow[t]{2}{*}{ TOTAL } & \multicolumn{4}{|c|}{$\begin{array}{l}\text { TOTAL APÓS } \\
\text { CRITÉRIOS DE } \\
\text { EXCLUSÃO }\end{array}$} & \multirow[t]{2}{*}{$\begin{array}{c}\text { ARTIGOS } \\
\text { DUPLICADOS }\end{array}$} & \multirow[t]{2}{*}{$\begin{array}{c}\text { ARTIGOS } \\
\text { SELECIONADOS }\end{array}$} \\
\hline & & 1 & 2 & 3 & 4 & & \\
\hline SCIENCEDIRECT & 624 & 544 & 234 & 2 & 2 & \multirow{2}{*}{0} & \multirow{2}{*}{5} \\
\hline SCOPUS & 75 & 62 & 35 & 5 & 3 & & \\
\hline
\end{tabular}

Fonte: Autores (2020).

No Quadro 3, está destacado os títulos dos trabalhos selecionados, a revista a qual foram publicados, os locais onde foram realizados os estudos, autores e o ano de publicação. Verifica-se que $80 \%$ dos trabalhos foram desenvolvidos na última década. 
Quadro 3 - Dados dos artigos selecionados.

\begin{tabular}{|c|c|c|c|}
\hline ARTIGO & REVISTA & LOCAL & AUTOR \\
\hline $\begin{array}{c}\text { Could city sewage sludge be directly used } \\
\text { into clay bricks for building construction? } \\
\text { comprehensive case study from Brazil }\end{array}$ & Journal of Building Engineering & Brasil & Areias et al. (2020) \\
\hline $\begin{array}{c}\text { Use of Sewage Sludge as Raw Material in } \\
\text { the Manufacture of Soft-Mud Bricks }\end{array}$ & $\begin{array}{c}\text { Journal of Materials in Civil } \\
\text { Engineering }\end{array}$ & Brasil & Ingunza et al. (2011) \\
\hline $\begin{array}{c}\text { Sewage sludge incorporating into fired clay } \\
\text { brick: Indoor air quality testing }\end{array}$ & $\begin{array}{c}\text { International Journal of } \\
\text { Innovative Technology and } \\
\text { Exploring Engineering }\end{array}$ & Malásia & Kadir et al. (2019) \\
\hline $\begin{array}{c}\text { Incorporation of Sewage Sludge in Clay } \\
\text { Brick and its Characterization }\end{array}$ & $\begin{array}{c}\text { Waste Management \& Research } \\
\text { Sludge valorization from wastewater } \\
\text { treatment plant to its application on the } \\
\text { ceramic industry }\end{array}$ & Malásia & Liew et al. (2004) \\
\hline
\end{tabular}

Fonte: Autores (2020).

Os artigos apresentados no Quadro 3 receberam uma avaliação inicial e são pertinentes para responder à questão levantada na organização do método P.I.C.O conforme a Tabela 1. A partir de então, foi realizado uma síntese das informações apresentadas nos estudos que levante a ideia da viabilidade técnica de se aplicar o lodo de ETE na fabricação de tijolos cerâmicos. As normas brasileiras foram utilizadas como comparativo para avaliar a relevância dos resultados.

\section{Resultados e Discussão}

\subsection{Composição química do lodo de ETE e da argila}

As características físicas, químicas e biológicas do lodo de ETE dependem do efluente de origem (local, tipo, tratamento e armazenamento), podendo ser mais ou menos tóxicos (Liew et al., 2004; Montero et al., 2009). Já a composição mineralógica da argila indica a sua condição de plasticidade, dependendo do tipo e da quantidade de argilominerais presentes, e de estabilidade conforme o seu nível de quartzo constituído (Ingunza et al., 2011).

Alguns óxidos são predominantes na composição química da argila e do lodo de ETE, apresentando-se então uma afinidade química e mineralógica entre os materiais estudados. Em quantidade relevante, são eles: $\mathrm{SiO}_{2}, \mathrm{Al}_{2} \mathrm{O}_{3}$ e $\mathrm{Fe}_{2} \mathrm{O}_{3}$. Todavia, foi identificado que a perda de ignição $(L O I)$ do lodo é bem maior do que a argila devido a presença de matéria orgânica (Areias et al., 2020).

A maioria dos lodos utilizados nas pesquisas eram oriundos de estações de tratamento de esgoto urbano (ETE) como pode ser verificado no Quadro 4. Já as argilas, foram coletadas nas próprias regiões onde os autores realizaram as pesquisas.

Quadro 4 - Origem do lodo de ETE por estudo.

\begin{tabular}{|l|c|}
\hline \multicolumn{1}{|c|}{ ORIGEM DO LODO } & ESTUDO \\
\hline Estação de tratamento de esgoto urbano. & Areias et al. (2020) \\
\hline Empresa de limpeza de fossa séptica com tratamento em lagoa de estabilização. & Ingunza et al. (2011) \\
\hline Estação de tratamento de esgoto urbano. & Kadir et al. (2019) \\
\hline Fossa séptica de instalações de tratamento de esgoto local. & Liew et al. (2004) \\
\hline Estação de tratamento de esgoto urbano. & Martínez-García et al. (2012) \\
\hline
\end{tabular}

Fonte: Autores (2020).

Para verificar a composição química dos materiais, os estudos fizeram análises por espectrometria de fluorescência $(X R F)$. Nos Quadros 5 e 6, observa-se as proporções semelhantes de $\mathrm{SiO}_{2}, \mathrm{Al}_{2} \mathrm{O}_{3}$ e $\mathrm{Fe}_{2} \mathrm{O}_{3}$ e outros resultados da caracterização dos materiais utilizados nas pesquisas levantadas, com exceção do trabalho de Liew et al. (2004) que não realizou esta análise. 
Quadro 5 - Composição química dos lodos utilizados para a fabricação de tijolos cerâmicos.

\begin{tabular}{|c|c|c|c|c|c|c|}
\hline \multicolumn{2}{|c|}{ ESTUDO } & \multirow{2}{*}{$\begin{array}{c}\text { Areias et al. (2020) } \\
8,83\end{array}$} & \multirow{2}{*}{$\frac{\text { Ingunza et al. (2011) }}{16,24}$} & \multicolumn{2}{|c|}{ Kadir et al. (2019) } & \multirow{2}{*}{$\frac{\text { Martínez-García et al. (2012) }}{20,33}$} \\
\hline \multirow{21}{*}{ 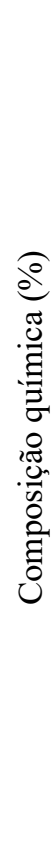 } & $\mathrm{Al}_{2} \mathrm{O}_{3}$ & & & 6,66 & 4,79 & \\
\hline & $\mathrm{SiO}_{2}$ & 14,26 & 33,89 & 14,30 & 16,30 & 46,37 \\
\hline & $\mathrm{Fe}_{2} \mathrm{O}_{3}$ & 7,78 & 12,89 & 9,85 & 9,35 & 8,55 \\
\hline & $\mathrm{K}_{2} \mathrm{O}$ & 0,71 & 1,50 & 0,71 & 1,07 & 3,25 \\
\hline & $\mathrm{MgO}$ & 0,76 & - & 1,15 & 0,85 & 2,19 \\
\hline & $\mathrm{MnO}$ & $<0,05$ & - & 0,60 & 0,50 & 0,28 \\
\hline & $\mathrm{Na}_{2} \mathrm{O}$ & 0,19 & - & 0,23 & - & 0,36 \\
\hline & $\mathrm{CaO}$ & 12,78 & 14,56 & 6,55 & 2,40 & 11,15 \\
\hline & $\mathrm{P}_{2} \mathrm{O}_{5}$ & 2,04 & - & 5,58 & 6,68 & 5,89 \\
\hline & $\mathrm{SO}_{3}$ & - & - & 9,20 & 4,61 & - \\
\hline & $\mathrm{TiO}_{2}$ & 0,63 & 2,87 & 0,50 & 0,52 & 0,85 \\
\hline & $\mathrm{BaO}$ & $<0,1$ & - & - & - & - \\
\hline & $\mathrm{CO}_{2} \mathrm{O}_{3}$ & $<0,1$ & - & - & - & - \\
\hline & $\mathrm{Cr}_{2} \mathrm{O}_{3}$ & $<0,1$ & - & - & - & - \\
\hline & $\mathrm{PbO}$ & $<0,1$ & - & - & - & - \\
\hline & $\mathrm{SrO}$ & $<0,1$ & - & - & - & - \\
\hline & $\mathrm{ZnO}$ & $<0,1$ & - & - & - & - \\
\hline & $\mathrm{ZrO}_{2}+\mathrm{HfO}_{2}$ & $<0,1$ & & - & - & - \\
\hline & $\mathrm{Zr}(\mathrm{ppm})$ & - & - & - & - & 161,7 \\
\hline & Outras & - & 16,59 & - & - & - \\
\hline & $L O I$ & 43,57 & - & - & - & 0,05 \\
\hline
\end{tabular}

Fonte: Autores (2020).

Quadro 6 - Composição química das argilas utilizadas para a fabricação de tijolos cerâmicos.

\begin{tabular}{|c|c|c|c|c|c|c|}
\hline \multicolumn{2}{|c|}{ ESTUDO } & \multirow{2}{*}{$\frac{\text { Areias et al. (2020) }}{21,23}$} & \multicolumn{2}{|c|}{ Ingunza et al. (2011) } & \multirow{2}{*}{$\frac{\text { Kadir et al. (2019) }}{18,40}$} & \multirow{2}{*}{$\frac{\text { Martínez-García et al. (2012) }}{12,13}$} \\
\hline \multirow{21}{*}{ 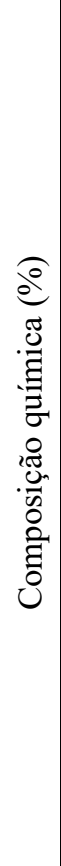 } & $\mathrm{Al}_{2} \mathrm{O}_{3}$ & & 23,01 & 20,47 & & \\
\hline & $\mathrm{SiO}_{2}$ & 62,98 & 68,68 & 55,32 & 49,30 & 55,82 \\
\hline & $\mathrm{Fe}_{2} \mathrm{O}_{3}$ & 2,73 & 3,12 & 7,40 & 6,78 & 4,83 \\
\hline & $\mathrm{K}_{2} \mathrm{O}$ & 1,21 & 2,42 & 5,82 & 3,09 & 2,78 \\
\hline & $\mathrm{MgO}$ & 0,48 & - & - & 0,80 & 1,49 \\
\hline & $\mathrm{MnO}$ & $<0,05$ & - & - & 0,4 & 0,03 \\
\hline & $\mathrm{Na}_{2} \mathrm{O}$ & 0,38 & - & - & - & 0,49 \\
\hline & $\mathrm{CaO}$ & 0,35 & 0,99 & 5,54 & - & 9,21 \\
\hline & $\mathrm{P}_{2} \mathrm{O}_{5}$ & 0,08 & - & - & - & 0,12 \\
\hline & $\mathrm{SO}_{3}$ & 0,89 & - & - & - & - \\
\hline & $\mathrm{TiO}_{2}$ & - & 0,97 & 0,78 & 0,94 & 0,83 \\
\hline & $\mathrm{BaO}$ & $<0,1$ & - & - & - & - \\
\hline & $\mathrm{Co}_{2} \mathrm{O}_{3}$ & $<0,1$ & - & - & - & - \\
\hline & $\mathrm{Cr}_{2} \mathrm{O}_{3}$ & $<0,1$ & - & - & - & - \\
\hline & $\mathrm{PbO}$ & $<0,1$ & - & - & - & - \\
\hline & $\mathrm{SrO}$ & 0,07 & - & - & - & - \\
\hline & $\mathrm{ZnO}$ & $<0,1$ & - & - & - & - \\
\hline & $\mathrm{ZrO}_{2}+\mathrm{HfO}_{2}$ & $<0,1$ & - & - & - & - \\
\hline & $\mathrm{Zr}(\mathrm{ppm})$ & - & - & - & - & 279,3 \\
\hline & Outras & - & 0,39 & 3,40 & - & - \\
\hline & $L O I$ & 9,47 & - & - & - & 10,55 \\
\hline
\end{tabular}

Fonte: Autores (2020).

Os Quadros 5 e 6 indicam que Martínez-García et al. (2012) utilizou o lodo e a argila que mais se aproximaram nas suas características de composição química. Isso aponta a possibilidade de substituir parcialmente o lodo de esgoto pela matéria-prima do tijolo cerâmico (Martínez-García et al., 2012).

$\mathrm{O}$ alto teor de $\mathrm{CaO}$ dos lodos apresentados no Quadro 5 pode ter sido devido os autores realizarem um tratamento 
prévio de inertização do lodo com cal virgem $-\mathrm{Ca}(\mathrm{OH})_{2}$ com o propósito de eliminar patógenos ou microrganismos conforme apontados por Areias et al. (2020). Já o baixo valor de LOI do lodo utilizados por Martínez-García et al. (2012) foi devido a uma secagem prévia realizada.

\subsection{Características do composto (lodo de ETE + argila)}

De acordo com a ABNT (2017), as propriedades físicas mínimas a serem analisadas em um tijolo cerâmico para atestar a sua conformidade devem ser a massa seca $\left(\mathrm{m}_{\mathrm{s}}\right)$ e o índice de absorção de água (AA). Já a propriedade mecânica é a resistência à compressão individual $\left(f_{\mathrm{b}}\right)$. Outros fatores que também auxiliam a verificar a qualidade de um tijolo são a retração linear, densidade aparente e perda de peso na ignição (LOI) (Liew et al., 2004; Martínez-García et al., 2012). Com relação a toxicidade final do tijolo referente a presença de metais pesados no lodo de ETE utilizados, alguns autores alertaram para a importância dessa verificação e realizaram a investigação através da análise de lixiviação.

\subsubsection{Proporção de mistura}

A proporção de mistura utilizada nos estudos foi a relação da porcentagem de lodo de ETE em relação a massa de argila a ser substituída no traço de fabricação do tijolo cerâmico. Para Martínez-García et al. (2012), a proporção de lodo é um fator chave para verificar as mudanças na qualidade dos tijolos produzidos.

No Quadro 7, verifica-se que todos os autores utilizaram a proporção de $0 \%$ (referência) para, assim, definir um tijolo padrão e poder comparar as suas características técnicas às demais proporções de mistura (Martínez-García et al., 2012). Além disso, entre os percentuais de mistura utilizados nas pesquisas (de $0 \%$ e $40 \%$ ), os mais aplicados foram $5 \%, 10 \%, 15 \%$ e $20 \%$.

Quadro 7 - Proporção de mistura de lodo em relação a massa de argila adotada pelos estudos.

\begin{tabular}{|c|c|c|c|c|c|c|c|}
\hline \multirow{2}{*}{\multicolumn{2}{|c|}{$\begin{array}{c}\text { PROPORÇÃO DE } \\
\text { MISTURA }\end{array}$}} & \multicolumn{5}{|c|}{ ESTUDOS } & \multirow{3}{*}{$\begin{array}{c}\text { QUANTIDADE } \\
\text { DE ESTUDOS } \\
\text { QUE } \\
\text { ADOTARAM } \\
5\end{array}$} \\
\hline & & \multirow{2}{*}{$\begin{array}{c}\begin{array}{c}\text { Areias et } \\
\text { al. } \\
(2020)\end{array} \\
\mathrm{X}\end{array}$} & \multirow{2}{*}{$\begin{array}{l}\begin{array}{l}\text { Ingunza et } \\
\text { al. }(2011)\end{array} \\
\mathrm{X} \\
\end{array}$} & \multirow{2}{*}{$\begin{array}{c}\text { Kadir et al. } \\
(2019) \\
X\end{array}$} & \multirow{2}{*}{$\begin{array}{c}\text { Liew et al. } \\
(2004) \\
X\end{array}$} & \multirow{2}{*}{$\begin{array}{c}\text { Martínez- } \\
\text { García et al. } \\
(2012)\end{array}$} & \\
\hline \multirow{12}{*}{ 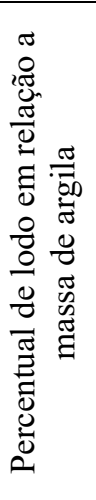 } & $0,0 \%$ & & & & & & \\
\hline & $1,0 \%$ & & & $\mathrm{X}$ & & $\mathrm{X}$ & 2 \\
\hline & $2,5 \%$ & $\mathrm{X}$ & & & & $\mathrm{X}$ & 2 \\
\hline & $5,0 \%$ & & $\mathrm{X}$ & $\mathrm{X}$ & & $X$ & 3 \\
\hline & $7,5 \%$ & & & & & $\mathrm{X}$ & 1 \\
\hline & $10 \%$ & $\mathrm{X}$ & $\mathrm{X}$ & $\mathrm{X}$ & $\mathrm{X}$ & $\mathrm{X}$ & 5 \\
\hline & $15 \%$ & $\mathrm{X}$ & $\mathrm{X}$ & & & $\mathrm{X}$ & 3 \\
\hline & $20 \%$ & & $\mathrm{X}$ & $X$ & $\mathrm{X}$ & & 3 \\
\hline & $25 \%$ & & $\mathrm{X}$ & & & & 1 \\
\hline & $30 \%$ & & $\mathrm{X}$ & & $\mathrm{X}$ & & 2 \\
\hline & $35 \%$ & & $\mathrm{X}$ & & & & 1 \\
\hline & $40 \%$ & & $\mathrm{X}$ & & $\mathrm{X}$ & & 2 \\
\hline \multicolumn{2}{|c|}{ TOTAL DE ADIÇÕES } & 4 & 9 & 5 & 5 & 7 & - \\
\hline
\end{tabular}

Fonte: Autores (2020).

Outrossim, o Quadro 7 expõe que o estudo de Ingunza et al. (2011) foi o que apresentou mais proporções de mistura. Para Areias et al. (2020), o limite de proporção para a fabricação do tijolo composto é de 50\% do lodo de ETE em relação a massa de argila, não considerando as características físicas e mecânicas resultantes da sua fabricação.

\subsubsection{Temperatura de sinterização}

A temperatura de queima influencia substancialmente na qualidade das propriedades mecânicas dos tijolos fabricados 
com argila (Pinheiro \& Holanda, 2010). A etapa de queima é essencial para a integração da argila com o lodo, desintegração efetiva da matéria orgânica e destruição dos patógenos, além de imobilizar produtos químicos (metais pesados) presentes no lodo (Monteiro et al., 2008; Weng et al., 2003).

As normas de tijolos cerâmicas não indicam uma temperatura mínima ou máxima de sinterização para a sua fabricação. De acordo com Monteiro et al. (2008), a temperatura usual para a fabricação de tijolos a base de argila está entre $700{ }^{\circ} \mathrm{C}$ e $1100{ }^{\circ} \mathrm{C}$. Logo, é observado no Quadro 8 que os autores utilizaram a temperatura de sinterização dentro desse intervalo, com frequência maior entre $950{ }^{\circ} \mathrm{C}$ e $1000^{\circ} \mathrm{C}$.

Quadro 8 - Temperatura de sinterização adotada pelos estudos para a fabricação dos tijolos.

\begin{tabular}{|c|c|}
\hline ESTUDO & TEMPERATURA $\left({ }^{\circ} \mathbf{C}\right)$ \\
\hline Areias et al. (2020) & 850 e 950 \\
\hline Ingunza et al. (2011) & 1000 \\
\hline Kadir et al. (2019) & 1050 \\
\hline Liew et al. (2004) & 985 \\
\hline Martínez-García et al. (2012) & 950 \\
\hline
\end{tabular}

Fonte: Autores (2020).

\subsubsection{Perda de peso na ignição}

Os estudos apresentaram um aumento na perda de peso na ignição associada ao acréscimo do percentual de lodo de ETE em relação a massa de argila presente na fabricação dos tijolos. Os autores concluíram que essa perda de peso é referente à combustão da matéria orgânica e à perda de umidade. O fato de aumentar a perda de peso de acordo com uma maior adição de lodo está relacionada a sua alta contribuição de matéria orgânica (Martínez-García et al., 2012).

\subsubsection{Densidade aparente}

Os resultados de densidade aparente após a queima (ver Quadro 9) indicaram uma proporção inversa ao acréscimo da porcentagem de lodo de ETE no traço de fabricação de tijolo, assim como na perda de peso na ignição, pois a densidade aparente depende diretamente da massa do tijolo. Logo, à medida que a quantidade de lodo aumenta na mistura, maior a perda de massa, criando-se assim vazios nas peças (Liew et al., 2004; Martínez-García et al., 2012).

Quadro 9 - Resultados de densidade aparente dos tijolos após a queima.

\begin{tabular}{|c|c|c|c|}
\hline \multirow{2}{*}{\multicolumn{2}{|c|}{$\begin{array}{l}\text { PROPORÇÃO DE } \\
\text { MISTURA }\end{array}$}} & \multicolumn{2}{|c|}{ DENSIDADE APARENTE $\left(\mathrm{g} / \mathrm{m}^{3}\right)$} \\
\hline & & \multirow{2}{*}{$\frac{\text { Liew et al. (2004) }}{2,560}$} & \multirow{2}{*}{$\frac{\text { Martínez-García et al. (2012) }}{1,615}$} \\
\hline \multirow{10}{*}{ 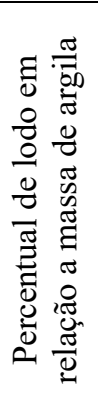 } & $0,0 \%$ & & \\
\hline & $1,0 \%$ & - & 1,600 \\
\hline & $2,5 \%$ & - & 1,546 \\
\hline & $5,0 \%$ & - & 1,423 \\
\hline & $7,5 \%$ & - & 1,410 \\
\hline & $10 \%$ & 2,430 & 1,409 \\
\hline & $15 \%$ & - & 1,340 \\
\hline & $20 \%$ & 2,348 & - \\
\hline & $30 \%$ & 2,275 & - \\
\hline & $40 \%$ & 2,220 & - \\
\hline
\end{tabular}

Fonte: Autores (2020).

\subsubsection{Absorção de água}

O parâmetro de absorção de água está diretamente associado a quantidade de poros nos tijolos produzidos após a 
queima, os quais, conforme foi analisado na densidade aparente, aumentam com o acréscimo de lodo de ETE inserido ao traço de fabricação. Para Areias et al. (2020) e Liew et al. (2004), a temperatura de sinterização também influencia na quantidade final de poros presentes na peça.

Níveis reduzidos de absorção de água em tijolos são um indicador de boa durabilidade para este material em relação a sua resistência às condições naturais do meio (Liew et al., 2004). De acordo com a NBR 15270-1, os resultados de absorção de água devem estar entre 8 e 25\% (linha tracejada vermelha na Figura 1) tanto para tijolos cerâmicos de vedação, quanto para tijolos cerâmicos estruturais (ABNT, 2017). Na Figura 1, visualiza-se os resultados dos artigos investigados que abordaram este parâmetro, considerando os índices em cada proporção de mistura adotada pelos autores.

Figura 1 - Absorção de água dos tijolos de argila com adição de lodo de esgoto em cada estudo.

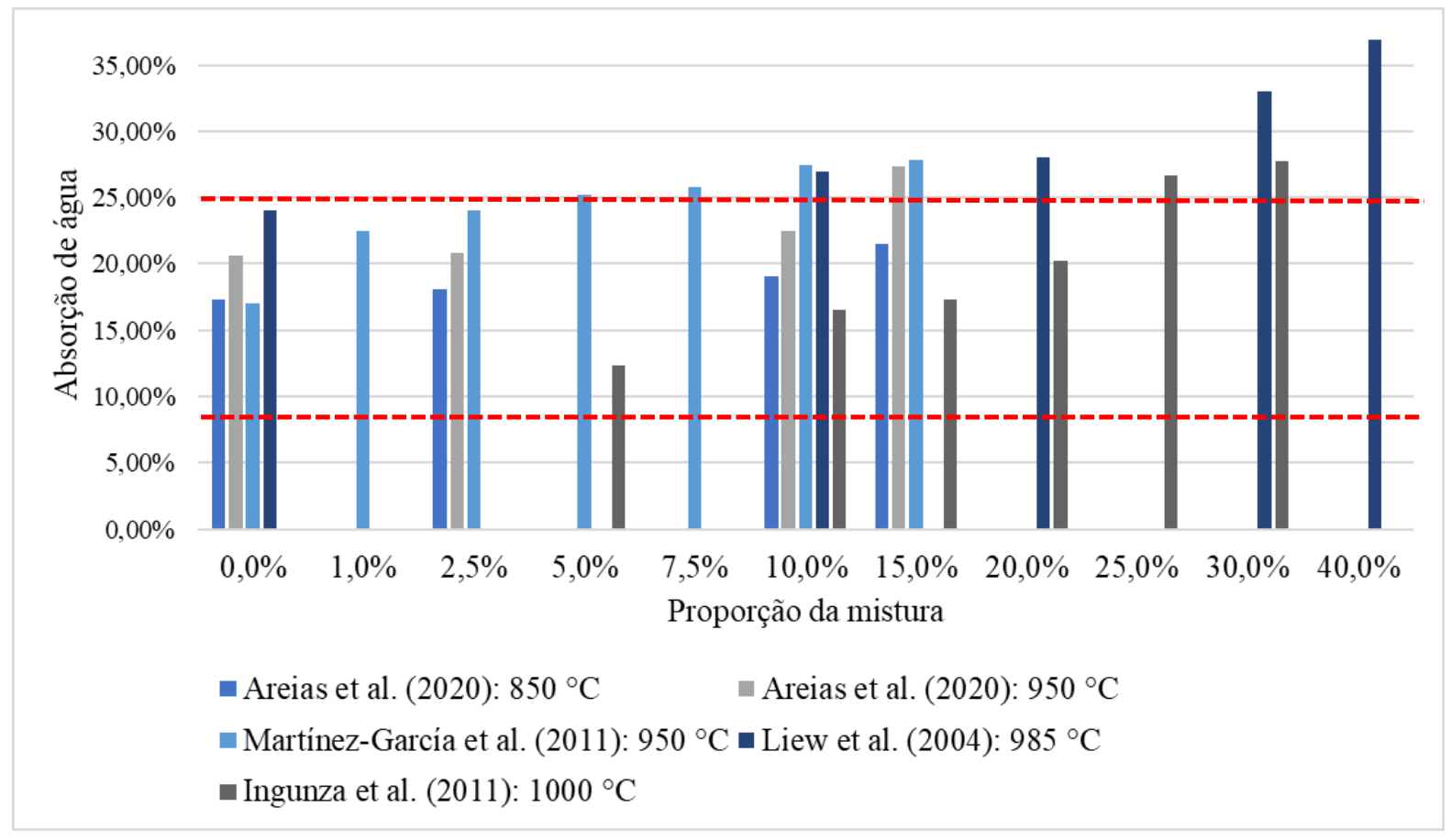

Fonte: Autores (2020).

Com isso, de acordo com a Figura 1, pode-se observar que 50\% dos tijolos avaliados em todas as pesquisas (exceto os traços referências) estão dentro do limite permitido pela ABNT (2017). Além disso, é visto que há uma tendência de aumento da absorção associado ao acréscimo de lodo na mistura. Conforme Areias et al. (2020) e Liew et al. (2004), o aumento da absorção de água é um indicativo para a redução da resistência mecânica do material.

\subsubsection{Retração linear}

É praticamente certo que ocorra a retração linear nos tijolos após os processos de queima (Liew et al., 2004). Quanto maior a retração, maior o potencial de surgir rachaduras ou deformações nas dimensões desses elementos que integram a construção de paredes (Areias et al., 2020).

De acordo com Martínez-García et al. (2012), os tijolos que tiverem retração linear abaixo de $8 \%$ apresentam uma boa qualidade. Na Figura 2, verifica-se que todos os estudos que avaliaram esse parâmetro apresentaram resultados bem abaixo deste limite, indicando que essa propriedade praticamente não é afetada pela adição do lodo de ETE na mistura de fabricação do tijolo. 
Figura 2 - Retração linear dos tijolos de argila com adição de lodo de esgoto em cada estudo.

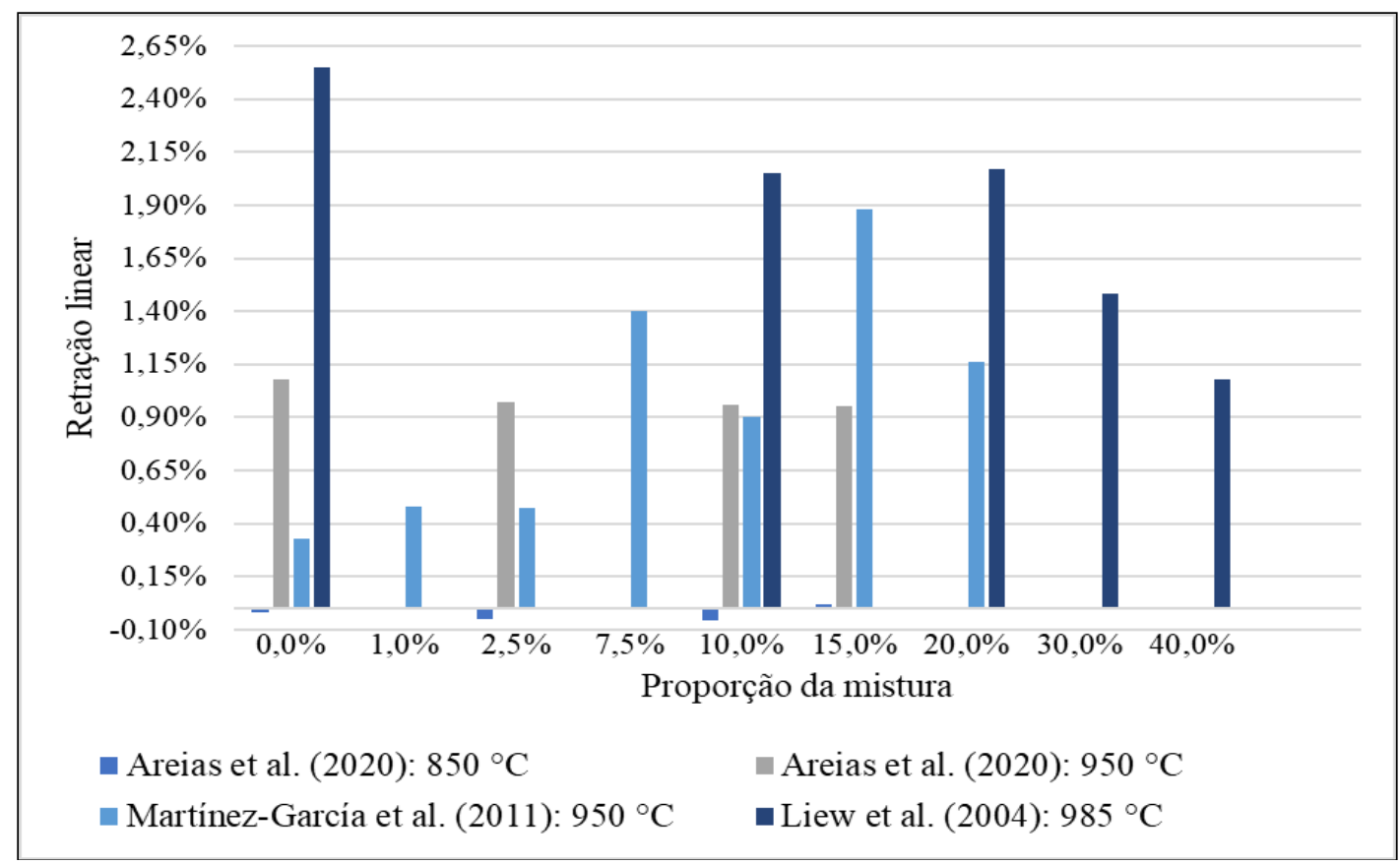

Fonte: Autores (2020).

É interessante destacar, de acordo com a Figura 2, que o estudo de Areias et al. (2020) utilizou duas temperaturas diferentes de sinterização e isso também resultou em valores bem distintos de retração, sendo então inferido uma tendência de aumento numérico do parâmetro com relação ao aumento da temperatura de produção, apesar da retração ainda continuar bem abaixo do limite de $8 \%$.

\subsubsection{Resistência mecânica}

No Brasil, não existe ainda uma recomendação ou exigência com relação a resistência mínima para utilização de tijolos com a adição de lodo de esgoto, pois não há uma normatização específica sobre isso. Entretanto, a norma ABNT NBR 15270-1, que trata das condições mínimas para a fabricação de tijolos cerâmicos, aponta a resistência mínima a compressão requerida $\left(f_{\mathrm{b}}\right)$ de 4,0 MPa (linha tracejada vermelha na Figura 3) para os que serão utilizados em paredes de vedação (ABNT, 2017). Nos artigos levantados, os autores utilizaram normas técnicas locais de fabricação de tijolo cerâmico para comparar os seus resultados.

O teste utilizado pelos estudos para verificar a resistência mecânica foi o ensaio de resistência à compressão. Verificase no Quadro 10 as dimensões dos corpos-de-prova utilizados nos ensaios e que não um padrão entre essas medidas, pois cada autor se baseou na normativa específica da região. 
Quadro 10 - Dimensões dos corpos-de-prova utilizados em cada estudo.

\begin{tabular}{|l|c|}
\hline \multicolumn{1}{|c|}{ ESTUDO } & $\begin{array}{c}\text { DIMENSÕES DOS CORPOS-DE-PROVA } \\
\text { (comprimento } \times \text { altura } \times \text { largura) }\end{array}$ \\
\hline Areias et al. (2020) & $115 \mathrm{~mm} \times 25 \mathrm{~mm} \times 10 \mathrm{~mm}$ \\
\hline Ingunza et al. (2011) & $220 \mathrm{~mm} \times 105 \mathrm{~mm} \times 45 \mathrm{~mm}$ \\
\hline Kadir et al. (2019) & $225 \mathrm{~mm} \times 125 \mathrm{~mm} \times 75 \mathrm{~mm}$ \\
\hline Liew et al. (2004) & $215 \mathrm{~mm} \times 102,5 \mathrm{~mm} \times 65 \mathrm{~mm}$ \\
\hline Martínez-García et al. (2012) & $60 \mathrm{~mm} \times 30 \mathrm{~mm} \times 10 \mathrm{~mm}$ \\
\hline
\end{tabular}

Fonte: Autores (2020).

Os resultados apresentados a seguir, na Figura 3, indicam que todos os estudos levantados tiveram ao menos um traço com argila e uma proporção de lodo que ultrapassou a resistência mínima requerida pela norma Brasileira. Ou seja, os tijolos fabricados pelos autores atendem a esta característica. Vale relembrar que cada estudo comparou os seus resultados com os requisitos de normas locais e que também apontaram conformidades.

Figura 3 - Resistência máxima à compressão do tijolo de argila com adição de lodo de esgoto em cada estudo.

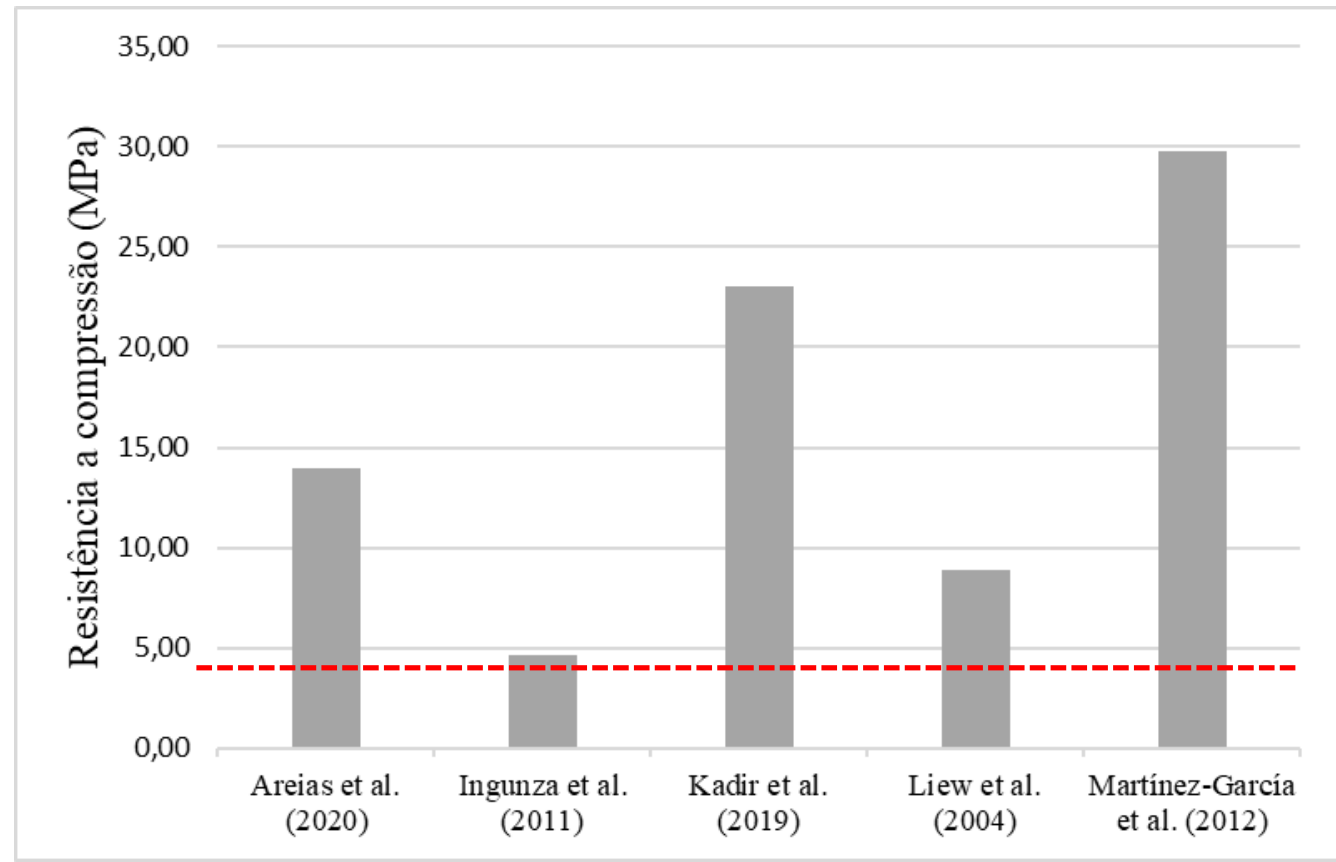

Fonte: Autores (2020).

Com isso, observa-se na Figura 3 que o estudo de Martínez-Garcia et al. (2012) atingiu o maior resultado de resistência a compressão e o de Ingunza et al. (2011) ficou praticamente no limite permitido pela ABNT NBR 15270-1.

As pesquisas apresentaram também uma relação inversa entre a resistência à compressão dos tijolos e a quantidade de lodo inserido no traço. Quanto maior era o percentual de lodo de ETE em relação a massa de argila no tijolo, menor seria o resultado da resistência. No Quadro 11, pode-se verificar o percentual e a temperatura de sinterização que proporcionaram as resistências máximas de cada estudo apresentada na Figura 3. 
Quadro 11 - Valores máximos de resistência à compressão em cada pesquisa.

\begin{tabular}{|l|c|c|c|}
\hline \multicolumn{1}{|c|}{ ESTUDO } & TEMPERATURA ( $\left.{ }^{\circ} \mathbf{C}\right)$ & $\begin{array}{c}\text { PROPORÇÃO DA } \\
\text { MISTURA }\end{array}$ & $\begin{array}{c}\text { RESISTÊNCIA À } \\
\text { COMPRESSÃO (MPa) }\end{array}$ \\
\hline Areias et al. (2020) & 950 & $2,50 \%$ & 14,00 \\
\hline Ingunza et al. (2011) & 1000 & $5,00 \%$ & 4,60 \\
\hline Kadir et al. (2019) & 1050 & $1,00 \%$ & 23,00 \\
\hline Liew et al. (2004) & 985 & $10,0 \%$ & 8,90 \\
\hline Martínez-García et al. (2012) & 950 & $2,50 \%$ & 29,80 \\
\hline
\end{tabular}

Fonte: Autores (2020).

Com isso, identifica-se no Quadro 11 que a proporção de mistura ideal dos tijolos para se alcançar a resistência a compressão do padrão normativo se encontra entre $1,00 \%$ e $10,0 \%$.

\subsubsection{Lixiviação de metais pesados}

A origem do lodo de ETE define a presença em maior ou menor quantidade de metais pesados que possam torná-lo tóxico para o ambiente humano, sendo as fontes industriais as que mais contribuem para isso (Jordán et al., 2005). É previsto que os contaminantes orgânicos presentes no lodo de ETE sejam destruídos durante a produção dos tijolos (de argila e lodo) devido a elevada temperatura de queima no seu processo de fabricação. Todavia, os metais pesados preocupam, pois não podem ser queimados e, assim, tem potencial de serem transferidos para o meio ambiente (Liew et al., 2004). Desta forma, este parâmetro é fundamental a ser analisado.

Os testes de toxicidade de metais pesados das pesquisas investigadas seguiram procedimentos padrões normativos de cada país ou região onde os estudos foram realizados, sendo citado no trabalho de Martínez-García et al. (2012) o método TCLP (Toxicity Characteristic Leaching Procedure) para avaliação deste parâmetro.

Os principais metais pesados levantados pelos trabalhos foram: arsênio, bário, cádmio, cromo, chumbo e prata. Os resultados dos estudos que investigaram a lixiviação de metais pesados (Ingunza et al., 2011; Liew et al., 2004; MartínezGarcía et al., 2012) apontaram que os tijolos cerâmicos com adição de proporções de lodo de ETE atendem as legislações vigentes em cada proporção de mistura utilizada e na região estudada. É notório que as caraterísticas de toxicidade são inerentes as regiões onde foram coletados os lodos utilizados nos trabalhos, podendo haver outros tipos de metais a serem investigados em outros locais de coleta.

\subsection{Oportunidades e lacunas}

A partir da revisão de literatura, pôde-se identificar algumas lacunas e oportunidades de pesquisa nos estudos investigados. Alguns temas são sugeridos: análise do ciclo de vida do produto, análise de viabilidade econômica, atestar a durabilidade, além de efeito da temperatura de queima.

A análise do ciclo de vida do produto poderá ser realizada para comparar os impactos ambientais dos tijolos feitos apenas com argila e os tijolos com adição parcial do lodo de ETE, pois não foi identificado um trabalho que evidenciassem a redução ou aumento desses impactos gerados com o aproveitamento deste resíduo de Estação de Tratamento de Esgoto na fabricação do tijolo cerâmico, sendo então uma pesquisa em potencial.

A análise de viabilidade econômica foi apresentada em apenas um estudo (Areias et al., 2020). Esta análise está adequada a realidade local (disponibilidade de recursos e equipamentos) onde foi realizada a pesquisa. Por tanto, novos estudos devem ser desenvolvidos para atestar a viabilidade econômica de se produzir o tijolo com argila e lodo de ETE, em cada região, em conjunto com a análise de viabilidade técnica para a proporção de mistura utilizada. 
Nenhum dos estudos investigados trabalhou com avaliação da durabilidade do tijolo cerâmico com adição de lodo de ETE no pós-construção, bem como a sua interação com outros materiais aplicados no processo de construção de uma parede.

Muitos autores apontaram para a influência da temperatura de sinterização nas propriedades dos tijolos cerâmicos, mas, apenas um estudo utilizou mais de uma temperatura para avaliar as características do tijolo com argila e adição de lodo de ETE. Além disso, foi destacado pelos pesquisadores que a temperatura de queima ideal não está bem definida.

\section{Conclusão}

Esta pesquisa mostrou que é viável tecnicamente produzir tijolos com argila e lodo de ETE na sua composição primária. Isso se deve principalmente à semelhança na composição química do lodo de ETE e da argila com proporções equivalentes principalmente de sílica, alumina, óxido de ferro e óxido de cálcio. Quanto mais próximo essas proporções, melhores são os resultados.

Como parâmetro de análise técnica, foi utilizado a normatização brasileira e pode-se atestar a viabilidade técnica dessa composição na fabricação de tijolos. Os estudos investigados se basearam em normas internacionais como parâmetro comparativo e afirmaram também a praticabilidade do uso de lodo de ETE compondo a matéria prima da fabricação de tijolos cerâmicos.

A temperatura de sinterização e a proporção de mistura que apresentaram os melhores resultados técnicos (dentro dos padrões normativos) foram respectivamente $950{ }^{\circ} \mathrm{C}$ e $2,50 \%$. Vale destacar que em um dos estudos, com adição de $10 \%$ de lodo de ETE, chegou-se a uma resistência relevante, apresentando um potencial de ser ainda mais sustentável. Mas, o seu resultado de absorção de água está além dos padrões previstos.

A pesquisa concluiu também que quanto maior a quantidade de lodo inserido na mistura de fabricação dos tijolos a base de argila, maior a sua porosidade no pós-queima. Com o aumento de poros, há, consequentemente, o aumento da absorção de água e redução da densidade aparente dos materiais produzidos com a mistura. Os resultados variaram de acordo com a composição química da argila e do lodo de ETE utilizados pelos autores.

Mais estudos são necessários para verificar a proporção ideal para a argila e o lodo utilizado em cada região, bem como a viabilidade econômica de se aplicar esses materiais a fim de serem comercializados efetivamente. Além disso, é importante estudar a durabilidade desse composto e a sua interação com outros materiais aplicados no assentamento e revestimento das paredes.

\section{Referências}

Areias, I. O. R., Vieira, C. M. F., Colorado, H. A., Delaqua, G. C. G., Monteiro, S. N., \& Azevedo, A. R. G. (2020). Could city sewage sludge be directly used into clay bricks for building construction? A comprehensive case study from Brazil. Journal of Building Engineering, $31,101374$.

Associação Brasileira de Normas Técnicas. (2017). NBR 15270-1: Componentes cerâmicos-Blocos e tijolos para alvenaria Parte 1: requisitos. ABNT.

Chang, Z., Long, G., Zhou, J. L., \& Ma, C. (2020). Valorization of sewage sludge in the fabrication of construction and building materials: A review. Resources, Conservation and Recycling, 154, 104606.

Cusidó, J. A., \& Cremades, L. V. (2012). Environmental effects of using clay bricks produced with sewage sludge: Leachability and toxicity studies. Waste management, 32(6), 1202-1208.

Esmeray, E., \& At1s, M. (2019). Utilization of sewage sludge, oven slag and fly ash in clay brick production. Construction and Building Materials, 194, 110121.

Feng, Y., Zhang, Y., Quan, X., \& Chen, S. (2014). Enhanced anaerobic digestion of waste activated sludge digestion by the addition of zero valent iron. Water research, 52, 242-250.

Ingunza, M. P. D., Duarte, A. C., \& Nascimento, R. M. (2011). Use of sewage sludge as raw material in the manufacture of soft-mud bricks. Journal of Materials in civil Engineering, 23(6), 852-856. 
Research, Society and Development, v. 10, n. 8, e22010817200, 2021

(CC BY 4.0) | ISSN 2525-3409 | DOI: http://dx.doi.org/10.33448/rsd-v10i8.17200

Jordán, M. M., Almendro-Candel, M. B., Romero, M., \& Rincón, J. M. (2005). Application of sewage sludge in the manufacturing of ceramic tile bodies. Applied Clay Science, 30(3-4), 219-224.

Kadir, A. A., Amirulamran, Salim, N. A., Hassan, M., \& Hashar, N. N. H. (2019). Sewage Sludge Incorporating Into Fired Clay Brick: indoor air quality testing. International Journal Of Innovative Technology And Exploring Engineering, 8(12), 1113-1118.

Koller, S. H., Couto, M. C. P. de P., \& Von Hohendorff, J. (2014). Manual de produção científica. Penso Editora.

Liew, A. G., Idris, A., Samad, A. A., Wong, C. H., Jaafar, M. S., \& Baki, A. M. (2004). Reusability of sewage sludge in clay bricks. Journal of Material Cycles and Waste Management, 6(1), 41-47.

Martínez-García, C., Eliche-Quesada, D., Pérez-Villarejo, L., Iglesias-Godino, F. J., \& Corpas-Iglesias, F. A. (2012). Sludge valorization from wastewater treatment plant to its application on the ceramic industry. Journal of environmental management, 95, 343-348.

Montero, M. A., Jordán, M. M., Hernández-Crespo, M. S., \& Sanfeliu, T. (2009). The use of sewage sludge and marble residues in the manufacture of ceramic tile bodies. Applied Clay Science, 46(4), 404-408.

Monteiro, S. N., Alexandre, J., Margem, J. I., Sánchez, R., \& Vieira, C. M. F. (2008). Incorporation of sludge waste from water treatment plant into red ceramic. Construction and Building Materials, 22(6), 1281-1287.

Pinheiro, B. C. A., \& Holanda, J. N. F. (2010). Efeito da temperatura de queima em algumas propriedades mecânicas de cerâmica vermelha. Cerâmica, 56(339), 237-243.

Santos, C. M. D. C., Pimenta, C. A. D. M., \& Nobre, M. R. C. (2007). A estratégia PICO para a construção da pergunta de pesquisa e busca de evidências. Revista Latino-Americana de Enfermagem, 15(3), 508-511.

Smol, M., Kulczycka, J., Henclik, A., Gorazda, K., \& Wzorek, Z. (2015). The possible use of sewage sludge ash (SSA) in the construction industry as a way towards a circular economy. Journal of Cleaner Production, 95, 45-54.

Souza, M. M. D., Anjos, M. A. S. D., Araújo, A. L. C., Soares, A. V. D. O., \& Souza, P. C. A. D. (2020). Uso do lodo de esgoto na produção de agregados leves: uma revisão sistemática de literatura. Matéria (Rio de Janeiro), 25(1).

Taki, K., Gahlot, R., \& Kumar, M. (2020). Utilization of fly ash amended sewage sludge as brick for sustainable building material with special emphasis on dimensional effect. Journal of Cleaner Production, 275, 123942.

Uggetti, E., Ferrer, I., Llorens, E., \& García, J. (2010). Sludge treatment wetlands: a review on the state of the art. Bioresource technology, 101(9), $2905-2912$.

Weng, C. H., Lin, D. F., \& Chiang, P. C. (2003). Utilization of sludge as brick materials. Advances in environmental research, 7(3), 679-685.

Yang, F., Zhou, W., Zhu, R., Dai, G., Wang, W., Wang, W., \& Wang, Z. (2021). Synergistic effects of amorphous porous materials and anhydrous $\mathrm{Na}_{2} \mathrm{CO}_{3}$ on the performance of bricks with high municipal sewage sludge content. Journal of Cleaner Production, 280, 124338.

Zhang, Q., Hu, J., Lee, D. J., Chang, Y., \& Lee, Y. J. (2017). Sludge treatment: current research trends. Bioresource technology, 243, $1159-1172$. 